\section{La relación entre la medicina y los proveedores médicos ${ }^{\star}$}

\author{
ANDRÉS HEERLEIN
}

\section{The relationship between medicine and medical suppliers}

In the last decades, many voices from academic medicine are questioning the relationship between the pharmaceutical industry or other medical suppliers and physicians. Conflict of interest has thus become a major issue of concern in medicine and is becoming increasingly important for medical journals and clinical work. Conflicts of interest may emerge in the daily practice of medicine, in medical and pharmacological research, in the process of publication of medical journals and in the dissemination of public and private medical information, such as conferences, lectures, books, pamphlets, monographs, etc. The credibility of physicians has been hampered in recent years by the proliferating connections between them and medical suppliers. The public seems to be increasingly skeptical of clinical medicine, since corporate actions placing profit over public health are usual. Research centers and projects as well as medical journals are considered an extension of the marketing arm of pharmaceutical companies. This undermines the credibility of medical practice, research work and pharmaceutical information, submitted to journals and later included in meta-analysis, influencing therapeutic guidelines. In the meantime, while disclosure has become standard practice in North America, it is not widely used in Latin-America and Europe. If independent researchers are financed by agencies not related to the industry, the problems caused by the increasing financial ties between the pharmaceutical industry and researchers could be overcome, avoiding conflicts of interest. Better disclosure policies and financial tie regulations are also urgently needed.

(Rev Med Chile 2014; 142: 361-367)

Key words: Clinical trials as topic; Conflict of interest; Disclosure; Ethics, medical; Social responsibility.

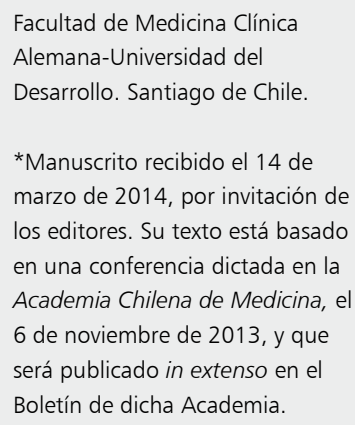

El autor declara no tener conflictos de intereses ni relación de dependencia con la Industria Farmacéutica $u$ otros proveedores médicos.
L a relación de la medicina y los proveedores médicos es amplia y extremadamente compleja. Es un tema muy relevante para la medicina actual y futura, que compromete los fundamentos básicos de la práctica médica, de la investigación clínica y la formación médica. En adelante, nos referiremos a la relación que existe entre la práctica de la medicina, la investigación clínica y la difusión del conocimiento con los proveedores médicos (industria farmacéutica, proveedores de equipos e implementos cardiológicos, quirúrgicos, etc). La relación entre los médicos y los proveedores médicos se ha caracterizado ge- neralmente por ser una relación de cooperación académico-profesional sinérgica. Los proveedores médicos participan muy activamente en las diversas actividades de las sociedades científicas, de universidades, hospitales, servicios de salud, clínicas privadas, etc., favoreciendo así la promoción y el desarrollo de la educación médica continua de pre y post-grado. Esta relación suele ser favorable para el desarrollo de la medicina, salvo cuando hay diferencias en los principios éticos, o surgen intereses discordantes o conflictivos.

La relación medicina-industria es estructural y funcionalmente asimétrica, tanto en sus objetivos 
como en sus funciones, que son muy diferentes. Es una relación basada en la cooperación profesional, la confianza y el respeto mutuo. El médico debe poder confiar en sus principales fuentes de información, incluidas las que provienen de los proveedores médicos. Los médicos recibimos frecuentemente la información que nos facilitan los proveedores, con plena confianza, y esto va a influir en las decisiones que finalmente tomemos. El ejercicio de la medicina apoyado por información errónea o carente de sustento científico tiene elevados índices de riesgo para los enfermos y para la salud pública.

La relación entre el médico y sus pacientes se caracteriza por su asimetría, ya que el médico ocupa siempre un rol dominante y posee gran parte del conocimiento. Esta asimetría es fundamental para el acto médico ya que exige y confiere al profesional un nivel superior y actualizado. Pero, simultáneamente, esta asimetría puede provocar grave perjuicio físico, cuando quien toma las decisiones terapéuticas ha recibido información errónea, sesgada o incompleta. Por ello es fundamental que el médico cuente siempre con información actualizada, veraz y confiable, basada en evidencia científica real, que garantice la calidad del proceso diagnóstico y terapéutico.

La profesión médica tiene como principio fundamental anteponer siempre los intereses de los pacientes a los intereses de los profesionales médicos u otros. Este principio vale para todo aquel que practique la medicina clínica, y está destacado en la gran mayoría de los textos de bioética y de ética médica ${ }^{1-3}$. Pero este principio, universalmente válido para toda práctica médica, no necesariamente debe ser válido para las empresas que actúan como proveedoras, cuyo principal objetivo -como toda empresa- es asegurar su propio desarrollo y maximizar los ingresos. $Y$ esto evidentemente no es objetable. Pero debemos reconocer que ambos principios e intereses son totalmente desiguales, y que pueden llegar a ser contrapuestos. Porque las empresas proveedoras tienen otro tipo de misiones, distintas a la de la profesión médica. Y también lo son sus fundamentos éticos. No son ni superiores ni inferiores, sino diferentes.

Uno de los problemas fundamentales que surgen en la relación de los médicos con la industria farmacéutica o con otros proveedores son los conflictos de intereses. Hablamos de conflicto de intereses "cuando una situación, donde un juicio o acción que debería estar determinado por un valor primario, definido por razones profesionales o éticas, puede estar o aparecer influido por un segundo interés". Este es el conflicto de intereses clásico que suele producirse al interior de la medicina y en la relación de la medicina con ciertas actividades comerciales.

\section{¿Cuáles son las principales fuentes de conflic- tos de intereses de la medicina?}

En primer lugar, surgen en la práctica diaria de la medicina, en las decisiones que el médico toma en su consulta, policlínico o pabellón. En segundo lugar, en la investigación médica, quirúrgica y farmacológica. También pueden surgir conflictos de intereses en la edición y publicación de revistas médicas. Finalmente, pueden surgir en la difusión de información médica que contenga algún tipo de información diagnóstica o terapéutica vinculada a los proveedores. Analicemos estos puntos:

\section{El ejercicio clínico de la medicina}

Es sabido que en la práctica médica diaria pueden surgir fácilmente conflictos de intereses en relación con los obsequios, invitaciones a congresos $\mathrm{u}$ otros que se ofrecen a los médicos, en la forma de abordaje del profesional, en la "entrega especial de muestras médicas", etc. Estos actos constituyen estrategias de marketing imprescindibles para las empresas proveedoras, destinadas a incrementar sus ventas. Ello no necesariamente debería originar conflictos de intereses, pero hay evidencias de que frecuentemente los ocasiona, amenazando el principio fundamental de la medicina de priorizar el interés del paciente. Recientemente la Cámara para la Innovación Farmacéutica (Chile), algunas compañías farmacéuticas internacionales y numerosas instituciones médicas nacionales e internacionales han intentado controlar este tipo de prácticas ${ }^{4-7}$. En los países desarrollados este tipo de conductas ha disminuido y sólo ocasionalmente se evidencian situaciones de conflictos de intereses.

Desde la perspectiva médica conviene recordar que este problema fue reconocido hace varias décadas y que se han realizado múltiples esfuerzos para intentar superarlo, a partir de iniciativas de los propios médicos y sus organizaciones, (como la declaración de ASOCIMED de 2005 o la reciente creación del grupo "Médicos sin Mar- 
ca"), quienes advirtieron precozmente que esta situación podía constituir una eventual amenaza para los principios básicos de la medicina y los fundamentos de la relación médico-paciente. La sola aceptación de obsequios onerosos, como viajes, invitaciones $\mathrm{u}$ otros, pueden determinar que el médico se vea expuesto, consciente o inconscientemente, a situaciones que lo conduzcan a un conflicto de intereses, especialmente cuando ostenta posiciones de liderazgo, poder o influencia. Los conflictos de intereses no sólo alteran la relación médico-paciente sino que deforman el acto médico, menoscabando la confianza del paciente, reduciendo la calidad diagnóstica y terapéutica o vulnerando la neutralidad y autonomía que debe existir en la toma de decisiones médicas. Asimismo, estas conductas pueden afectar las funciones docentes y formativas de los médicos, quienes necesariamente transmiten mucha información y conocimiento a sus pares y alumnos. El rol docente del médico requiere de una total independencia de intereses o compromisos con proveedores, y debe constituir un ejemplo de idoneidad para sus alumnos.

Hay indicios que sugieren que, en los últimos años, algunos proveedores médicos han intentado restringir sus estrategias de marketing con el cuerpo médico. No obstante, hasta hace pocos meses ciertas compañías insistían en mantener un marketing agresivo. Un ejemplo reciente lo constituye la estrategia diseñada por una de las mayores empresas farmacéuticas multinacionales para acelerar su crecimiento en la República Popular China, publicado en The Lancet ${ }^{8}$. Para aumentar rápidamente sus ventas esta empresa decidió otorgar invitaciones u obsequios a los médicos chinos que cooperaban con ella. Se destinaron más de US\$ 600 millones para financiar viajes, regalos o incluso pagos directos a los galenos chinos. Se utilizaron agencias de viajes como mediadoras para pagar a los médicos que prescribieran fármacos de esa empresa. Hace pocos meses, enfrentados a la autoridad china, varios directivos de dicha empresa admitieron haber recurrido a estas prácticas, según informó el Ministerio de Seguridad Pública del país asiático. Cuatro ejecutivos de la multinacional están detenidos en ese país mientras dura el proceso judicial. Hace pocas semanas el mismo laboratorio farmacéutico manifestó su intención de cambiar radicalmente su estrategia de marketing en el ámbito médico.

\section{Conflictos de intereses en investigación clínica y farmacológica}

El primer ensayo clínico aleatorio en la historia de la medicina es el del Streptomycin Trial Committee, que en 1948 analizó la eficacia de la estreptomicina en el tratamiento de la tuberculosis, marcando ese año como el comienzo de una nueva era en la medicina moderna ${ }^{9}$. Ese estudio fue financiado con fondos fiscales, por lo que se inscribió en la historia como uno de los primeros ensayos clínicos independientes. Posteriormente, la gran demanda de conocimiento farmacéutico/ terapéutico provocó un progresivo acercamiento entre la investigación clínica de la medicina académica tradicional y la industria farmacéutica. Surgieron así los primeros estudios privados, dependientes de un financiamiento externo proporcionado por los proveedores médicos. Estos son denominados ensayos clínicos dependientes, ligados a la industria farmacéutica o a otras entidades comerciales. En un principio esta colaboración dio buenos resultados, con novedosos aportes al conocimiento farmacológico. Posteriormente, la presión por generar "nuevo conocimiento" se fue intensificando, y la colaboración científica entre industria y medicina académica se fue debilitando. Los proveedores comenzaron a organizar sus propios ensayos de investigación farmacológica, con operadores e investigadores externos, no siempre vinculados al mundo académico. La principal consecuencia de este fenómeno fue un aumento notorio de los estudios y publicaciones dependientes del financiamiento privado. A partir de la década de 1990-99 los ensayos clínicos dependientes comenzaron a institucionalizarse en la forma de estudios multicéntricos transnacionales, llegando a constituir una verdadera red internacional de ensayos clínicos, dependientes de un financiamiento privado y controlados por operadores externos. En la actualidad, gran parte de la información clínico-farmacológica se genera en este nivel, ligando así el ámbito de los proveedores médicos con la medicina clínica.

Uno de los problemas frecuentes de este tipo de ensayos clínicos es que muchos tratamientos aparentemente eficaces no revelan mayor efectividad en la práctica médica real. Eficacia es diferente de efectividad y se limita principalmente al área de los ensayos clínicos controlados. Los estudios de eficacia terapéutica intentan dilucidar las dosis óptimas, la respuesta clínica máxima y la tolerabi- 
lidad de los tratamientos; generalmente se realizan en poblaciones seleccionadas y depuradas, por lo que no siempre entregan información representativa de la práctica médica real. Los estudios de efectividad, por otra parte, suelen aportar mayor información acerca de lo que ocurre en la realidad clínica.

En nuestro país la investigación farmacológica auspiciada por la industria (ensayos clínicos dependientes) está en franco crecimiento. Se estima que anualmente se invierten más de 30 millones de dólares en este tipo de investigación y que existe una clara tendencia a potenciarla. Muchos simpatizantes de este proceso hablan de un notable desarrollo de la investigación científica nacional. Sin embargo, conviene señalar que este tipo de investigación es, en estricto rigor, sólo un proceso de evaluación tecnológica y no una investigación científica original. En los ensayos clínicos sólo se verifican las características de ciertos productos, pero no se genera conocimiento nuevo. Además, gran parte de los datos que se recogen a través de estos estudios no permanece en el país de origen, sino que migra al extranjero, porque son estudios multicéntricos internacionales dependientes de las empresas que los financian.

Otro problema es que las vías de financiamiento de estos ensayos no siempre son conocidas, tanto en su origen como en su destino, y suele ser difícil obtener información precisa y confiable acerca de los destinatarios finales.

Finalmente, se han reportado problemas en el diseño de estos estudios, como por ejemplo problemas con el tratamiento posterior al ensayo clínico, o con la brusca interrupción de los mismos; ambas situaciones pueden provocar graves descompensaciones de los sujetos incorporados al estudio, recaídas o síntomas de descontinuación. Además, se han reportado problemas con el consentimiento informado de pacientes discapacitados, con la confidencialidad que todo paciente merece, con la confiabilidad y el ocultamiento de los datos, con múltiples otros dilemas éticos $y$, finalmente, con el quiebre de la confianza del paciente por su médico.

Algunos autores han llamado la atención a la baja frecuencia de reportes negativos en los ensayos dependientes. Se ha sugerido la presencia de un eventual 'sesgo positivo' relacionado con la fuente de financiamiento de los ensayos clínicos, y se han publicado estudios con desiguales resultados.
R. A. Davidson revisó 107 ensayos clínicos de diferentes patologías de Medicina Interna, encontrando que en la mayoría de ellos había una correlación significativa entre la fuente de financiamiento y los resultados del ensayo clínico ${ }^{10}$. Un estudio observacional de 370 ensayos clínicos aleatorios incluidos en los meta-análisis de la Cochrane Library reveló diferencias significativas entre los ensayos dependientes e independientes, especialmente cuando se les compara según la magnitud del financiamiento externo declarado ${ }^{11}$.

En Cardiología, Ridker et al. confirmaron que los ensayos clínicos revelan una significativa mayor frecuencia para reportar resultados favorables al promotor del proyecto, mientras que los ensayos financiados por instituciones independientes reportan resultados y conclusiones más cercanas a la realidad clínica cotidiana ${ }^{12}$.

En el ámbito de la Psiquiatría y farmacoterapia, se han publicado recientemente dos grandes estudios independientes realizados en los Estados Unidos de Norteamérica, destinados a evaluar la efectividad de los antipsicóticos y de los antidepresivos, en un contexto más cercano al mundo real. Uno de ellos, el estudio CATIE (Clinical Antipsychotic Trials of Intervention Effectiveness) sobre la efectividad de los agentes antipsicóticos, no logró establecer diferencias de efectividad entre los antipsicóticos atípicos noveles y un antipsicótico clásico: la perfenazina ${ }^{13}$. En cuanto a los antidepresivos, el estudio secuencial para el tratamiento de las depresiones, el $\operatorname{STAR}^{\star} \mathrm{D}$, no demostró diferencias entre los distintos antidepresivos, como sugerían diferentes ensayos clínicos dependientes; tampoco se observaron ventajas entre diferentes estrategias de tratamiento para las depresiones refractarias ${ }^{14}$. En una revisión del tema, publicada en el año 2008, analizamos y comparamos las fortalezas y debilidades de diferentes estrategias de investigación terapéutica, ofreciendo una visión global de las diversas formas, ya sea la de los ensayos clínicos dependientes (financiados por los proveedores médicos internacionales) o de los proyectos de investigación independientes de los proveedores médicos ${ }^{15}$.

En conclusión, resulta indispensable que los futuros ensayos clínicos cuenten con la misma neutralidad, transparencia e independencia que requiere cualquier proyecto de investigación médica. La selección de las muestras y la publicación de los datos debe ser confiable, con una metodo- 
logía consensuada y ampliamente probada, con la autorización y vigilancia de un comité de ética debidamente acreditado y con claros mecanismos de descarte de eventuales conflictos de intereses. Esto es especialmente válido para los ensayos conducidos por personas naturales o por instituciones privadas, que no sólo están dirigiendo el estudio sino que también están percibiendo honorarios por participar en los ensayos. Es recomendable que no sólo en estos casos sino que en todos los ensayos clínicos financiados por los proveedores médicos, sean farmacológicos o de otra naturaleza, se realicen esfuerzos para evitar o minimizar sesgos asociados a un eventual conflicto de intereses ${ }^{16}$.

Un ejemplo que ilustra lo grave que puede llegar a ser el tema de las relaciones comerciales entre los médicos y la industria en los ámbitos de la investigación farmacológica fue el caso del Rofecoxib, un antiinflamatorio introducido hace algunos años en todo el mundo pero que ya no existe. Este fármaco antiinflamatorio fue lanzado al mercado mundial alrededor del año 2001 por una empresa multinacional, con gran difusión y un aparentemente "sólido respaldo científico". Poco tiempo después de su lanzamiento se reconocieron en los Estados Unidos de Norteamérica casos de accidentes cardiovasculares fatales relacionados con el Rofecoxib, que llevaron a la suspensión internacional de la venta de este fármaco. Tanto médicos como pacientes comenzaron a preguntarse cómo era posible que estos efectos cardiotóxicos no se hubieran pesquisado durante los ensayos clínicos realizados previamente, necesarios para lograr la aprobación por parte de la Food and Drug Administration (FDA) de los Estados Unidos de Norteamérica. Algunos familiares de las víctimas del Rofecoxib en los Estados Unidos de Norteamérica acudieron a los tribunales de justicia, demandando a la empresa farmacéutica correspondiente. Durante el proceso judicial una comisión de médicos expertos demostró que se había incurrido en faltas muy graves, tanto por parte de la industria como por algunos médicos. Por ejemplo, se constató que el laboratorio en cuestión había utilizado los nombres de autores "invitados", para redactar y publicar los estudios clínicos sobre Rofecoxib. A su vez, estos autores, que figuraban y se presentaron ante la FDA como los investigadores responsables, en realidad no habían visto nunca a los pacientes y no habían realizado personalmente los trabajos que habían suscrito. Las revistas especializadas habían publicado esos estudios confiando en la veracidad de los datos y en la reputación de los falsos autores. Este lamentable caso fue publicado en el año 2008 en el Journal of the American Medical Association (JAMA) ${ }^{17}$.

\section{Los proveedores y las publicaciones médicas}

$\mathrm{Al}$ analizar las principales revistas y las publicaciones sobre ensayos clínicos controlados financiados por la industria, es frecuente observar una baja prevalencia de reportes adversos o una total omisión de resultados negativos o desfavorables al fármaco estudiado. Frecuentemente los resultados de eficacia favorecen al producto en estudio mientras que el perfil de efectos colaterales o indeseados es mínimo. Ocasionalmente estos resultados nunca alcanzan la luz pública, sino que se retienen en su fuente. Se ha descrito una contradicción entre los ensayos clínicos dependientes y los independientes, sugiriendo la presencia de un sesgo en la información proveniente de algunos ensayos clínicos financiados, lo que podría incidir en las prácticas clínicas y en las decisiones de los médicos de todo el mundo. Así lo entienden Ross et al en un artículo del JAMA, señalando que la información médica sesgada o de menor confiabilidad aumenta la probabilidad del error médico, disminuyendo la calidad y la efectividad de la acción terapéutica ${ }^{17}$.

Diversos autores han sugerido que los resultados sesgados o las conclusiones parciales provenientes de algunos estudios dependientes han influido negativamente sobre la calidad y la confiabilidad de muchas guías clínico-terapéuticas basadas en evidencia ${ }^{18-21}$. Cabe destacar que los principales criterios, contenidos y conductas recomendados por estas pautas terapéuticas (guías clínicas y manuales terapéuticos), rigen las políticas de salud de muchos países. Por lo tanto, las consecuencias finales de este proceso no sólo amenazan la calidad y confiabilidad del trabajo médico sino que trascienden las fronteras de la relación médico-paciente, pudiendo afectar aspectos políticos y económicos de relevancia en salud pública.

Es importante que información promocional por parte de los proveedores se base en publicaciones veraces y científicamente sustentadas. En el año 2011 un prestigioso laboratorio multi- 
nacional promocionó en los Estados Unidos de Norteamérica un fármaco antidepresivo como agente afrodisíaco, sin contar con la evidencia científica necesaria. La FDA castigó esta conducta con una multa de tres mil millones de dólares. Es posible que en el futuro estos castigos ejemplares inhiban las conductas de promoción que carezcan de un fundamento sólido, alejadas de la realidad y de la evidencia científica en medicina. En síntesis, las conclusiones de numerosos trabajos sobre los ensayos clínicos coinciden en que las futuras investigaciones sobre la calidad de los tratamientos médicos deberían evitar los estudios con eventuales sesgos, considerando sólo ensayos clínicos objetivos y transparentes, prefiriendo los ensayos clínicos independientes destinados a aportar información sólida y confiable acerca de los complejos aspectos de la terapia médica.

\section{¿Que ha ocurrido en nuestro país en la última década en esta materia?}

En el año 2005 la Asociación de Sociedades Científicas Médicas de Chile (ASOCIMED) acordó en asamblea extraordinaria firmar un documento de recomendaciones para evitar conflictos de intereses en medicina (Tabla 1). A partir de la publicación de estas recomendaciones, ASOCIMED participó en múltiples foros, mesas redondas y conferencias sobre el tema ${ }^{6,7}$.

En el año 2008, la Cámara de Innovación Farmacéutica de Chile (CIF) -que reúne a la industria farmacéutica internacional en Chile- acordó internamente un código de ética que regula y controla las conductas que pueden originar estos conflictos. Desde entonces numerosos laboratorios farmacéuticos han cambiado su conducta frente a

\section{Tabla 1. Recomendaciones de la Asociación de Sociedades Científicas Médicas de Chile para las relaciones entre sus socios y la industria proveedora}

Las Sociedades Médicas-Científicas miembros de ASOCIMED se comprometen a difundir entre sus socios y a apoyar entre los especialistas las siguientes recomendaciones:

1. Aceptar la menor cantidad posible de obsequios de los proveedores médicos y no aceptar obsequios físicos de un valor superior a los US \$100.- por año

2. Aceptar como máximo el financiamiento parcial o total de un viaje nacional y un viaje internacional al año, y sólo cuando es realmente un viaje educativo o académico

3. Sólo aceptar un viaje o un obsequio cuando esté asegurada la exclusión de todo tipo de compromiso, vínculo, obligación o relación profesional con el patrocinador o financista del regalo

4. Evitar toda participación activa o pública en eventos de difusión médica, cuyo control organizador dependa de proveedores médicos o donde pueda verse lesionada la neutralidad y autonomía científica

5. Abstenerse de participar en proyectos de investigación financiados por la Industria Farmacéutica u otro proveedor médico, en los que se perciban ingresos personales. Se recomienda participar sólo en trabajos clínicos donde los fondos o ingresos se dirigen a instituciones sin fines de lucro, como p.ej., las Sociedades Científicas

6. Evitar todo tipo de exámenes, interconsultas, derivaciones u otros que puedan constituir conflicto de intereses

7. Mantener siempre una actitud neutral, transparente y ética en la relación médico-paciente y con los proveedores

8. No aceptar ni recibir cualquier índole de pagos de parte de los proveedores que pueda significar connivencia comercial en la atención profesional

9. Evitar la promoción pública en los medios que pueda establecer algún vínculo con los proveedores

10. Evitar todo tipo de vínculo comercial, directo o indirecto, con los proveedores médicos

11. Declarar antes de cada presentación pública, conferencia, clase, etc. todos los vínculos comerciales que se hayan tenido en los últimos 3 años con los proveedores médicos

12. Evitar participar en cualquier situación académica o editorial donde se pudiera producir un eventual conflicto de intereses

13. Favorecer siempre una conducta médica ética, transparente y distanciada de cualquier forma de conflicto de intereses 
los médicos. En agosto de 2013 la CIF y el Colegio Médico de Chile firmaron un acuerdo de "Marco Ético de Relaciones", por medio del cual ambas instituciones se comprometen a velar para que el actuar entre los profesionales de la medicina y los laboratorios asociados a la CIF se desarrolle en un marco de pleno cumplimiento de las disposiciones éticas que ambas organizaciones han definido. Este acuerdo tiene como principal objetivo hacer más transparente la relación entre los médicos y los proveedores médicos.

\section{Referencias}

1. Council on Ethical and Judicial Affairs, American Medical Association. Conflicts of interest. Physician ownership of medical facilities. JAMA 1992; 267: 2366-9.

2. Roa A. Etica y bioética. Editorial Andrés Bello, 1998.

3. Goic A. La responsabilidad profesional del médico. En "El Fin de la Medicina", Editorial Mediterráneo, 2000.

4. World Medical Association. Guidelines on the Relationship between Physicians and commercial Enterprises, 2004. Disponible en http://www.wma.net/e/press/2004.

5. Wazana A, Primeau F. Ethical considerations in the relationship between physicians and the pharmaceutical industry. Psychiatr Clin N Am 2002; 25: 647-63.

6. Heerlein A. Recomendaciones para un control de los conflictos de interés en medicina. Rev Chil NeuroPsiquiatría 2005; 43: 83-7.

7. Armas R, Heerlein A. Conflictos de Intereses y trabajo clínico. Rev Chil Pediatr 2005; 76: 237-9.

8. Editorial. Doctors and pharma in China. The Lancet 2013; 382 (9887): 102-3.

9. Demirdjian G. Historia de los ensayos clínicos aleatorizados. Arch Argent Pediatr 2006 ; 104 (1) : 52-61.

10. Davidson RA. Source of funding and outcome of clinical trials. J Gen Intern Med 1986; 1: 155-8.

11. Als-Nielsen B, Chen W, Gluud C, Kjaergard LL. Association of funding and conclusions in randomized drug trial: a reflection of treatment effect or adverse events. JAMA 2003; 290: 921-8.

12. Ridker P, Torres J. Reported outcomes in major cardiovascular clinical trials funded by for-profit and notfor-profit organizations: 2000-2005. JAMA 2006; 295: 2270-4.

13. Swartz M, Stroup Ts, Mcevoy J, Davis S, et al. What CATIE found: results from the schizophrenia trial. Psychiatric Services 2008; 59: 500-6.

14. Warden D, Rush AJ, Trivedi MH, Fava M, Wisniewski SR. The $\mathrm{STAR}^{\star} \mathrm{D}$ Project results: a comprehensive review of findings. Curr Psychiatry Rep 2007; 9 (6): 449-59.

15. Heerlein A. Los Ensayos Terapéuticos y la Práctica Clínica Real. Vertex 2009; 20: 107-19.

16. Heerlein A. What is the impact of financial conflicts of interests on the development of psychiatry? World Psychiatry 2007; 6: 36-7.

17. Ross JS, Hill KP, Egilman DS, Krumholz HM. Guest authorship and ghostwriting in publications related to rofecoxib: a case study of industry documents from Rofecoxib litigation. JAMA 2008; 299 (15): 1800-12.

18. Bhandari M, Busse JW, Jackowski D, Montori VM, Schünemann H, Sprague S, et al. Association between industry funding and statistically significant pro-industry findings in medical and surgical randomized trials. CMAJ 2004; 170: 477-80.

19. Fava GA. A different medicine is possible. Psychother Psychosom 2006; 75: 1-3.

20. Hirsch L. Randomized clinical trials: what gets published, and when? CMAJ 2004; 170: 481-3.

21. Ioannidis JP. Interpretation of tests of heterogeneity and bias in meta-analysis. J Eval Clinical Pract 2008; 14: 9517. 